\title{
Implementation of a Rayleigh Scattering Optical Depth Parameterization
}

\author{
Susan R. Kemball-Cook \\ Keith E. Grant
}

Global Climate Research Division, L-262

Lawrence Livermore National Laboratory

P.O. Box 808, Livermore, CA 94551

October 1994

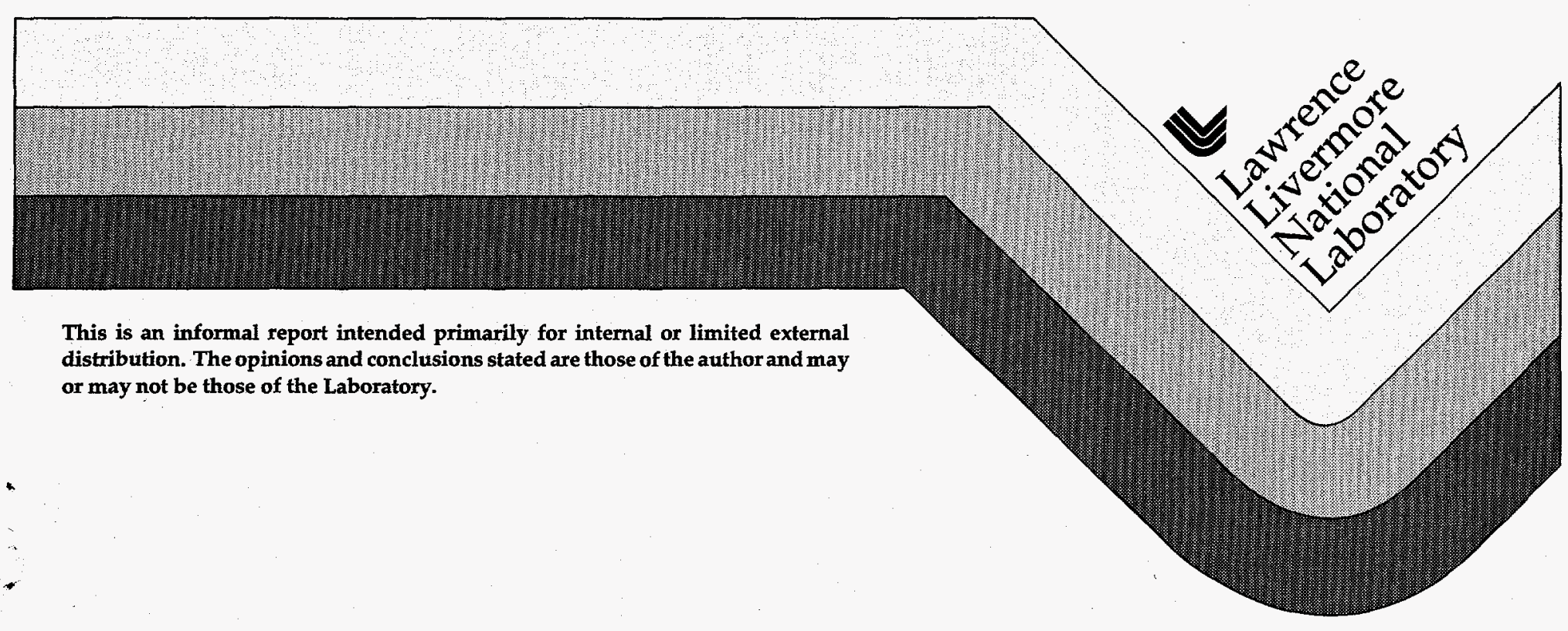




\section{DISCLAIMER}

This document was prepared as an account of work sponsored by an agency of the United States Government. Neither the United States Government nor the University of California nor any of their employees, makes any warranty, express or implied, or assumes any legal liability or responsibility for the accuracy, completeness, or usefulness of any information, apparatus, product, or process disclosed, or represents that its use would not infringe privately owned rights. Reference herein to any specific commercial product, process, or service by trade name, trademark, manufacturer, or otherwise, does not necessarily constitute or imply its endorsement, recommendation, or favoring by the United States Government or the University of California. The views and opinions of authors expressed herein do not necessarily state or reflect those of the United States Government or the University of California, and shall not be used for advertising or product endorsement purposes. 


\section{DISCLAIMER}

Portions of this document may be illegible in electronic image products. Images are produced from the best available original document. 
Implementation of a Rayleigh scattering Optical Depth Parameterization

Susan R. Kembal1-Cook

Reith E. Grant

DisTR,Bunow of This DOCUMENT is UNimiteo 
A new procedure for calculating Rayleigh scattering cross sections and optical depths has been developed for use in the solar radiation transfer model of the LLNL Chemistry-Radiation-Transport Models. This procedure replaces the previous method for determining the optical depths in which the parameterization of Hansen and Travis (1974) was used to generate a table of optical depths for wavelengths ranging from $0.135 \mu \mathrm{m}$ to $0.729 \mu \mathrm{m}$; the table was then placed directly in the shortwave package. Hansen and Travis calculate Rayleigh scattering optical depths, $\tau$, using

$$
\tau=0.08569 \lambda^{-4}\left(1+0.0113 \lambda^{-2}+0.00013 \lambda^{-4}\right)
$$

where $\lambda$ is wavelength in $\mu \mathrm{m}$ and the standard surface pressure is $1013 \mathrm{mB}$.

The new scheme uses instead the parameterization of Nicolet (1984) to find the Rayleigh scattering cross sections for an arbitrary set of wavelength bins, and from these cross sections, determines the optical depths. The Nicolet parameterization was recommended by the WMO (1986), and calculates Rayleigh scattering cross sections using

$$
\sigma_{R S}=\frac{4.02 \times 10^{-28}}{\lambda^{-(\varsigma-x)}}
$$

where $x=0.389 \lambda+0.09426 / \lambda-0.3328$ for $\lambda<0.55 \mu \mathrm{m}$ and $\mathrm{x}=0.04$ for $\lambda>0.55 \mu \mathrm{m}$.

The average Rayleigh scattering cross section for a wavelength bin extending from $\lambda_{1}$ to $\lambda_{2}$ is found by integrating (2) across the bin

$$
\left\langle\sigma_{R S}\right\rangle=\left(\lambda_{2}-\lambda_{-}\right)^{-1} \int_{\lambda_{I}}^{\lambda_{z}} \frac{4.02 \times 10^{-28}}{\lambda^{-\left(4-x_{i}\right.}} d \lambda
$$

Since there is no obvious closed form for the solution to (3), an eight point Gaussian quadrature was used to evaluate the integral. From the cross sections obtained in this manner, the corresponding Rayleigh scattering optical depths to the bottom of the atmosphere, $\tau_{\mathrm{RB}}$, were calculated using

$$
\left\langle\tau_{R E}\right\rangle=\left\langle\sigma_{R S}\right\rangle \int_{z}^{\infty} n(z) d z .
$$

$\left\langle\tau_{R B}\right\rangle$ is the average of $\tau_{R B}$ across the wavelength bin, and $n(z)$ is the total number of absorbing molecules in a column of height $z$. $n(z)$ is found by

$$
\int_{0}^{\infty} n(z) d z=-\int_{P_{3}}^{C}\left(\frac{N_{5}}{M_{A} g}\right) d P \approx \frac{N_{0} P_{0}}{M_{A} g}
$$

where $\mathrm{N}_{0}$ is Avogadro's number, $\mathrm{M}_{\mathrm{A}}$ is the molecular weight of air, $g$ is the acceleration of gravity, and $P_{0}$ is the surface pressure. 
The accuracy of the Nicolet scheme was tested by comparing the Nicolet optical depths for a data set containing 126 new wavelength bins to the optical depths produced by the Hansen and Travis parameterization for those same bins. The optical depth data table from an earlier LLNL CRT Model consisted of 148 bins and their corresponding optical depths and was generated from (1). An interpolation was performed which mapped these 148 old wavelength bins and $\tau_{\mathrm{RB}}$ onto the 126 new bins. Then, Rayleigh scattering cross sections and $\tau_{\mathrm{RB}}$ were calculated for the new bins using the Nicolet parameterization. A comparison of the two sets of optical depths for the new wavelength bins is shown in figure 1.

The two sets of $\tau_{\mathrm{RB}}$ agree well; the percentage difference between the two curves never grows larger than 10\%. (See figure 2). The region of greatest deviation occurs at $0.2-0.4 \mu \mathrm{m}$, where $\tau_{\mathrm{RB}}$ changes most rapialy.

The Nicolet optical depths were then compared to optical depths produced by using higher order Gaussian quadratures to solve (3). A quadrature of order 16 was used as a baseline to compare with quadratures of lower orders; it was assumed that the integral would have converged for quadrature orders lower than 16.

For the one point quadrature, the largest value of the percent difference from the

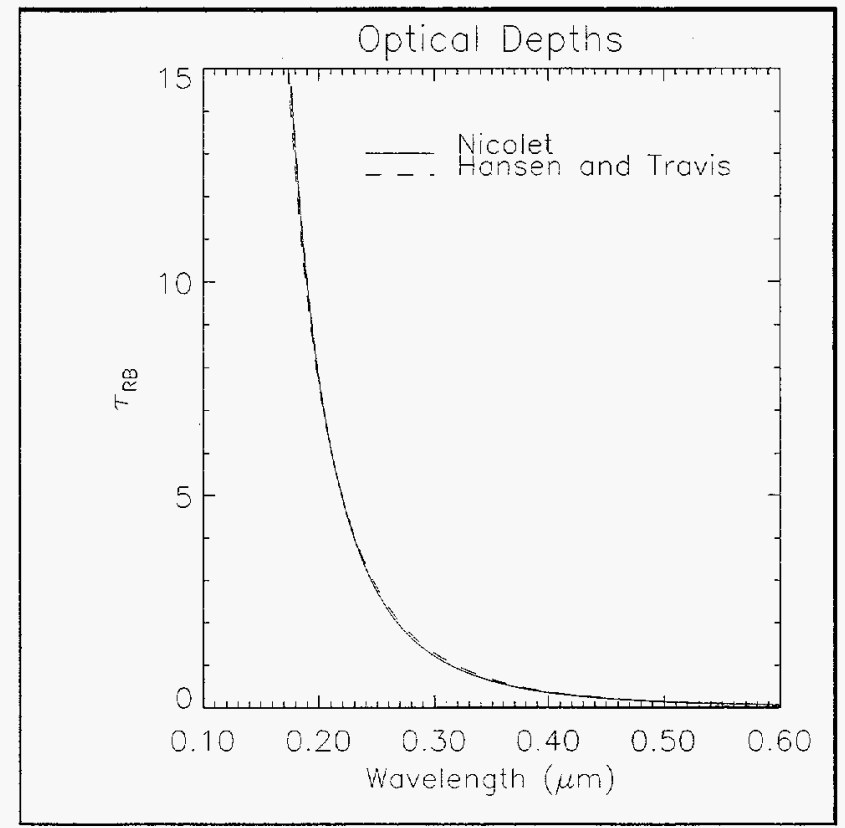

Figure 1 Optical Depth Comparison 16 point quadrature is $-0.25 \%$.

(See figure 3). For the two point cases the value of the maximum difference drops to $-1.7 \times 10^{-6} \%$, and for the four and eight point cases, the maximum differences are $5.5 \times 10^{-8} \%$ and $1.9 \times 10^{-8} \%$, respectively. It is reasonable to assume that the eight point quadrature is sufficiently accurate for use in evaluating (3), as the values for the quadratures have converged by the time we reach the 8 point case.

The shape of the percent difference curves is determined in part by the structure of the wavelength bins. Bin spacing increases from $1.55 \times 10^{-3} \mu \mathrm{m}$ at $0.176 \mu \mathrm{m}$ to $4.95 \times 10^{-3} \mu \mathrm{m}$ at $0.303 \mu \mathrm{m}$, and has a constant bin spacing of $5.0 \times 10^{-3} \mu \mathrm{m}$ for $\lambda>0.303 \mu \mathrm{m}$. In all cases, the percent difference between a given quadrature order and order 16 increases as the bin spacing increases, and then declines as the bin spacing becomes constant for $\lambda>0.303 \mu \mathrm{m}$. The peaks in the percent difference curves near $0.303 \mu \mathrm{m}$ are caused by the rapid change of $\tau_{\mathrm{RB}}$ in this region; the approximation to the integral has its lowest accuracy here.

The one point quadrature consistently underestimates the value 
of $\tau_{\mathrm{RB}}$, and, as might be expected of a linear approximation, errs most where the $\tau_{\mathrm{RB}}$ curve deviates most strongly from linearity. The two point case shows a similar behavior. The four and eight point quadratures overestimate the values of $\tau_{R B}$, but show the same shape curve as the one and two point cases.

In conclusion, the Nicolet parameterization successfully calculates $\tau_{\mathrm{RB}}$ for an arbitrary set of wavelength bins when the integral (3) is evaluated using an eight point Gaussian quadrature.

The Nicolet $\tau_{\mathrm{RB}}$ agree well with those of Hansen and Travis for the case tested here.

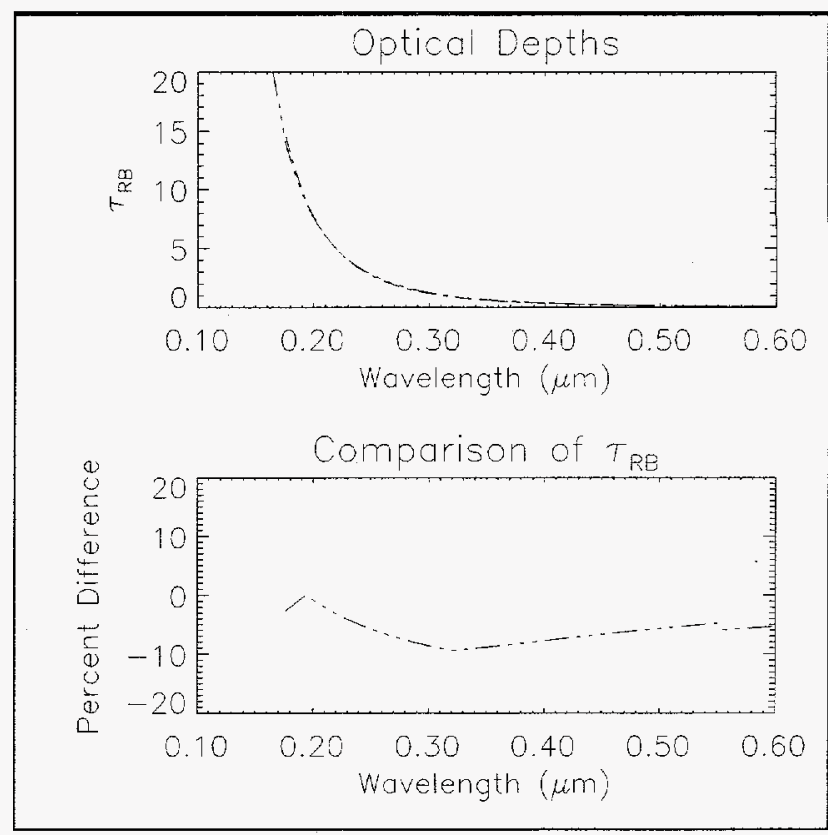

Figure 2 Percent Difference Curves

Acknowledgement

Work performed under the auspices of the U.S. Department of Energy by the Lawrence Livermore National Laboratory under contract No. W-7405-Eng-48 and was supported in part by both the Department - Energy Office of Environmental Analysis, the U.S. Environmental Protection Agency, and the Carbon Dioxide Research Program.

\section{$\underline{\text { References }}$}

Hansen, J. and Travis,I. 1974: Light scattering in planetary atmospheres. Space Science Rev. 16, 14.

Nicolet, M. 1984: On the molecular scattering in the terrestrial atmosphere: an empirical formula for its calculation in the homosphere. Planet. Space Sci. 32, 1467.

WMO, 1986. Atmospheric Ozone 1985. Report 16. 

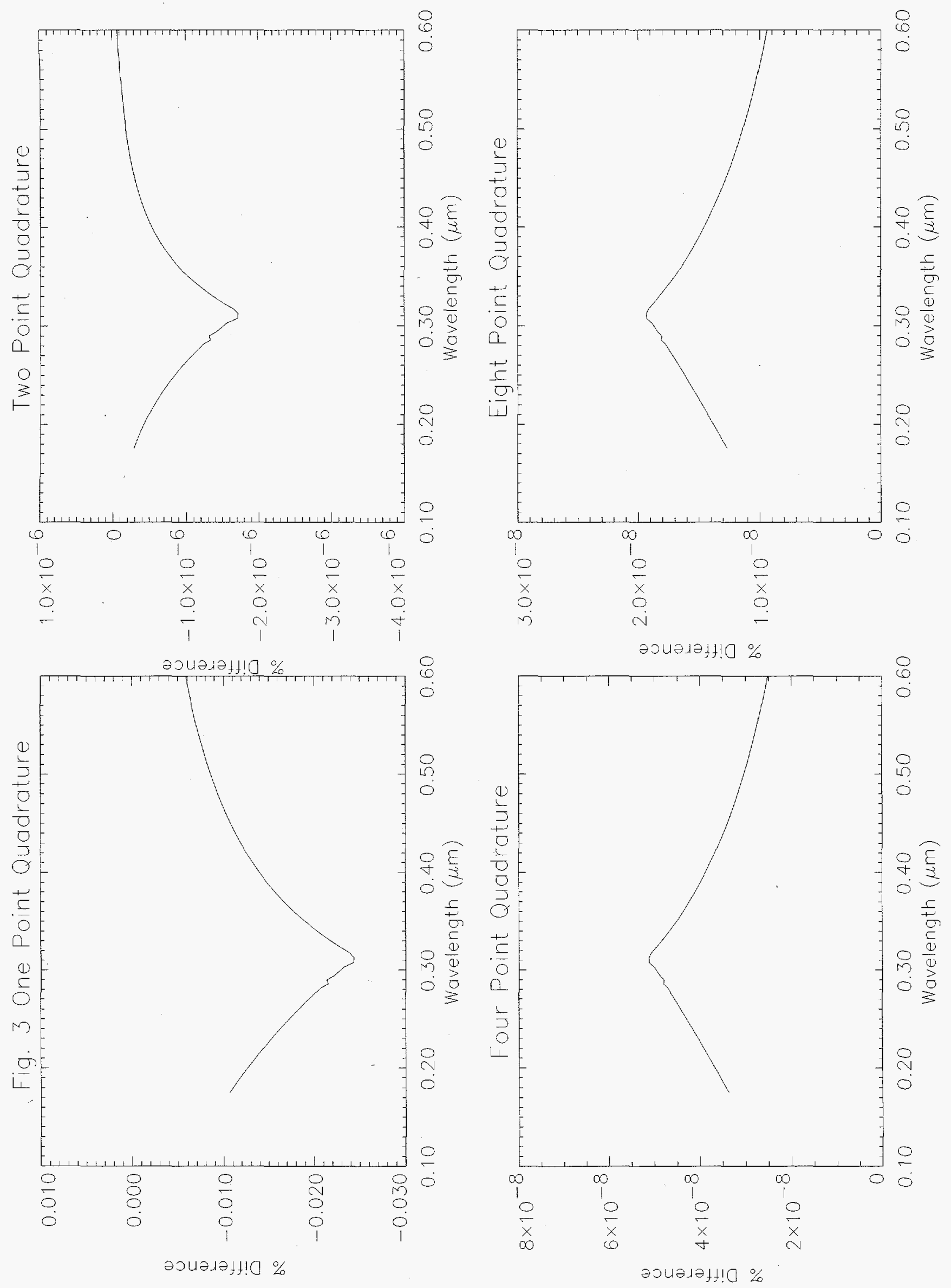